\title{
Composición de vinos tintos elaborados por procedimientos alternativos de vinificación
}

\section{Composition of red wines made by alternative winemaking procedures}

\author{
G. González-Neves, G. Favre y D. Piccardo
}

Facultad de Agronomía, Universidad de la República. Avda. Garzón 780. C.P. 12900, Montevideo, Uruguay

\begin{abstract}
Resumen. Los procedimientos empleados en la vinificación pueden atenuar el impacto de eventos climáticos extremos, que afectan la calidad de los vinos. El objetivo de este trabajo fue comparar una vinificación por maceración tradicional (MT) con una vinificación con extracción diferida de antocianos y maceración extendida (EDA+ME), en seis variedades de Vitis vinifera L. (Tannat, Cabernet Sauvignon, Merlot, Cabernet Franc, Syrah y Marselan). Los vinos fueron analizados a los 6 meses de la vinificación. Los vinos de Marselan y Cabernet Sauvignon tuvieron $\mathrm{pH}$ muy elevados. Los vinos con mayores contenidos fenólicos totales fueron Tannat y Marselan, que también tuvieron los mayores contenidos de antocianos y proantocianidinas. La mayor intensidad colorante se obtuvo en los vinos Marselan y la menor en Syrah. Los vinos producidos con las dos técnicas presentaron diferencias en tonalidad y \% de amarillo (mayores en EDA+ME) y rojo (mayores en MT). Los vinos MT tuvieron mayores contenidos de antocianos y taninos más polimerizados. Los vinos EDA + ME tuvieron contenidos significativamente mayores de polifenoles totales, catequinas y mayor reactividad de los taninos. Un Análisis Discriminante Canónico confirmó que la variedad de uva fue el factor que incidió en mayor medida en las características de los vinos.
\end{abstract}

\begin{abstract}
Winemaking procedures can mitigate the impact of extreme climate events that affect the quality of wines. The aim of this work was to compare traditional maceration winemaking (TM) with those made with delayed extraction of anthocyanins and extended maceration (EDA+EM), in six varieties of Vitis vinifera $\mathrm{L}$. (Tannat, Cabernet Sauvignon, Merlot, Cabernet Franc, Syrah and Marselan). The wines were analyzed after 6 months of winemaking. Marselan and Cabernet Sauvignon wines had very high $\mathrm{pH}$ values. Tannat and Marselan wines had the highest total phenolic, anthocyanins and proanthocyanidins contents. The highest colorant intensity was obtained in Marselan and the lowest in Syrah wines. The wines produced with the two techniques presented differences in the average values of hue and \% of yellow (higher in DEA+EM) and red (highest in TM). TM wines had higher anthocyanin contents and more polymerized tannins than DEA+EM wines. The wines DEA+EM had significantly higher total polyphenols and catechins contents, and higher reactivity of the tannins than TM wines. By means of a Canonical Discriminant Analysis it was verified that the grape variety was the factor that had the greatest impact on the characteristics of the wines.
\end{abstract}

\section{Introducción}

Las características de un vino dependen de factores ambientales, entre los que se destacan suelo y clima, factores genéticos, entre los cuales es relevante la variedad de uva, y factores humanos, que incluyen las prácticas vitícolas y enológicas adoptadas.

Los procedimientos de vinificación pueden tener un fuerte impacto sobre las características de los vinos. El contacto entre las partes sólidas de la uva y el líquido permite la extracción de polifenoles durante la maceración, desarrollada en simultáneo con la fermentación alcohólica en la vinificación en tinto clásica o tradicional.

Los pigmentos extraídos de la uva y los que se originan durante la vinificación, conservación y crianza determinan el color de los vinos tintos. Los antocianos participan en reacciones de condensación y adición con los taninos y con compuestos que se producen en la fermentación alcohólica (como el ácido pirúvico y el acetaldehido). Los compuestos generados tienen color diferente y mayor estabilidad que los pigmentos originales [1-3]. Las altas concentraciones de antocianos y taninos favorecen las condensaciones entre ambos y la posterior formación de pigmentos poliméricos [1-4].

En la fase pre-fermentativa y en las maceraciones cortas se prioriza la extracción de los antocianos, que son fácilmente solubles en agua, en relación con la de los taninos, poco solubles en medios acuosos y más solubles en presencia de etanol. En las maceraciones largas (extendidas) se prolonga el contacto entre las partes sólidas de la uva y el vino en la etapa post-fermentativa, con lo que se promueve la extracción de taninos de las semillas 
y su condensación con los antocianos [1-4]. Todos estos compuestos pueden volver a fijarse en los hollejos y las semillas, si el contacto con éstos es prolongado $[5,6]$.

Los factores fundamentales que afectan la extracción de polifenoles de hollejos y semillas, y las reacciones en las que los mismos participan, son la temperatura, la acidez y la concentración de etanol del medio $[1,5,7]$.

El clima incide de manera fundamental sobre la composición de la uva y las características de los vinos. En el caso de Uruguay, la variabilidad climática se ha incrementado, con períodos recurrentes de lluvias intensas, que en muchos años determinan problemas de sanidad y/o de una menor calidad enológica de las uvas. Estos problemas tienen consecuencias negativas en la calidad de los vinos, afectando entre otros aspectos a la intensidad y estabilidad del color de los vinos tintos.

Numerosas alternativas de vinificación han sido propuestas para mejorar la extracción de pigmentos y la estabilidad del color de los vinos tintos. El objetivo de la mayor parte de estos procedimientos alternativos es modificar las condiciones de extracción de los distintos polifenoles de la uva y las relaciones entre estos compuestos y los que se producen en la fermentación alcohólica, procurando incidir en las reacciones entre ellos [1]. Entre estos procedimientos se encuentra la llamada extracción diferida de antocianos, que procura demorar la difusión de los componentes de los hollejos en el mosto y suministrar menores cantidades de oxígeno en las primeras etapas de la vinificación, de manera de proteger particularmente a los antocianos de los fenómenos de oxidación [8]. A su vez, el retraso en la extracción de estos compuestos busca promover las reacciones con metabolitos de las levaduras y la condensación con taninos, que generan pigmentos más estables en el tiempo [8,9].

Por otra parte, las maceraciones post-fermentativas, en las que se prolonga el contacto entre las partes sólidas de la uva y el vino, procuran una mayor extracción de taninos de las semillas, promoviendo sus condensaciones con los antocianos [1].

El objetivo de este trabajo fue evaluar los efectos de procedimientos alternativos de vinificación sobre el color y la composición polifenólica de vinos tintos de las variedades Merlot, Cabernet Franc, Cabernet Sauvignon, Syrah, Marselan y Tannat. Se comparó una maceración tradicional (MT) con una alternativa que combinó una extracción diferida de antocianos y una extracción diferida $(\mathrm{EDA}+\mathrm{ME})$. De esta manera se procuró modificar las relaciones entre antocianos y taninos, al modificar la extracción de componentes de los hollejos en la etapa prefermentativa y primeras fases de la fermentación alcohólica, y realizar una etapa de maceración postfermentativa más larga.

\section{Materiales y métodos}

Se elaboraron vinos de las variedades Tannat Merlot, Cabernet Franc, Marselan, Cabernet Sauvignon y Syrah. Los vinos de cada variedad fueron elaborados mediante una vinificación con extracción diferida de antocianos y maceración extendida (EDA+ME), como alternativa a la vinificación por maceración tradicional (MT).

Se emplearon $70 \mathrm{~kg}$ de uva en cada vinificación, que se realizó con dos repeticiones por técnica. Las uvas fueron cosechadas en su madurez tecnológica (valores máximos de azúcares y mínimos de acidez total) y transportadas en cajones plásticos de $20 \mathrm{~kg}$. En la bodega fueron procesadas con una descobajadota-moledora Alfa $60 \mathrm{R}$ (Italcom, Italia) y se depositaron en tanques de acero inoxidable de 100 litros de capacidad. Se realizó una prueba de homogeneidad de los mostos depositados en los 4 tanques que se emplearon por ensayo, verificando que las densidades de los mismos no presentaran diferencias.

Posteriormente se sembraron levaduras secas activas Natuferm 804 (OenoBioTech, Francia), en una dosis de 15 gramos por hectolitro. Se agregaron 5 gramos de anhídrido sulfuroso cada $100 \mathrm{~kg}$ de uva. Las temperaturas de fermentación fueron controladas entre 27 y $30^{\circ} \mathrm{C}$.

La duración del encubado para la maceración tradicional (MT) se definió en función de los potenciales fenólicos de las uvas (datos no mostrados). Las maceraciones se realizaron durante 7 días en Merlot, Cabernet Sauvignon, Cabernet Franc y Syrah, y 8 días en Tannat y Marselan. Durante las maceraciones se realizaron dos remontajes por día, dos de bazuqueos hasta hundir el "sombrero". En las elaboraciones por EDA+ME se postergó el inicio de los remontajes hasta que el mosto en fermentación contenía aprox. 5\% de etanol, y se mantuvo el encubado en la fase post-fermentativa, con una duración total de 15 días en todas las variedades.

En todos los mostos se controlaron la densidad y la temperatura dos veces por día, para verificar el desarrollo de las fermentaciones alcohólicas. Luego del descube, los orujos fueron prensados con una prensa manual de acero inoxidable y se juntaron los jugos de prensa y de gota. Los vinos se guardaron en recipientes de vidrio de 10 litros hasta el momento de su análisis.

Los vinos fueron analizados a los 6 meses de la vinificación, evaluando su composición básica, color y composición polifenólica. La composición básica se evaluó analizando los contenidos de alcohol, acidez total y volátil, $\mathrm{pH}$ y azúcares reductores, empleando un analizador de infrarrojo Winescan TM Autosampler 79000 (Foss, USA) y el software Foss Integrator versión 154 (Foss, Dinamarca).

El color fue evaluado mediante los índices enológicos tradicionales de Glories [10] y los parámetros CIELAB, de acuerdo con Ayala et al. [11].

La composición polifenólica global se estimó empleando los índices espectrofotométricos clásicos. Los polifenoles totales se determinaron con el reactivo de Folin Ciocalteu, según Singleton y Rossi [12]; los antocianos totales según Ribéreau-Gayon y Stonestreet [13]; las catequinas a través del método de Swain y Hillis [14] y las proantocianidinas de acuerdo con Ribéreau-Gayon y Stonestreet [15]. La reactividad de los taninos fue evaluada mediante el índice DMACH (reacción con el p-dimetilamino cinamaldehído), analizado según Vivas et al. [16]. El índice de polimerización de los taninos se calculó relacionando el DMACH con la concentración de proantocianidinas (DMACH/LA), de acuerdo con Vivas et al. [16].

Se realizaron dos repeticiones de cada análisis por muestra, luego de centrifugar los vinos a 3500 r.p.m. durante 3 minutos, empleando una centrífuga CENCOM II (Selecta, España).

Todas las determinaciones se hicieron con un espectrofotómetro Cole Parmer S2100UV ${ }^{+}$(Cole Parmer, USA), empleando celdas de vidrio de $1 \mathrm{~mm}$ de recorrido 
Tabla 1. Valores medios \pm desviaciones estándar de la composición general de los vinos. Los valores seguidos por una misma letra no presentaron diferencias en el test de Tukey al $5 \%$.

\begin{tabular}{|cccc|}
\hline & Grado alcohólico & Acidez total & pH \\
\hline Variedad & & & \\
\hline Syrah & $11.79 \pm 0.17 \mathrm{~b}$ & $2.67 \pm 0.11 \mathrm{~b}$ & $3.67 \pm 0.04 \mathrm{e}$ \\
Cab. Franc & $12.68 \pm 0.19 \mathrm{a}$ & $2.48 \pm 0.05 \mathrm{~b}$ & $3.90 \pm 0.03 \mathrm{c}$ \\
Merlot & $12.44 \pm 0.18 \mathrm{a}$ & $2.68 \pm 0.07 \mathrm{~b}$ & $3.82 \pm 0.02 \mathrm{~d}$ \\
Cab. Sauv. & $11.32 \pm 0.13 \mathrm{c}$ & $2.57 \pm 0.08 \mathrm{~b}$ & $4.05 \pm 0.01 \mathrm{~b}$ \\
Marselan & $12.66 \pm 0.07 \mathrm{a}$ & $2.67 \pm 0.11 \mathrm{~b}$ & $4.17 \pm 0.02 \mathrm{a}$ \\
Tannat & $12.62 \pm 0.16 \mathrm{a}$ & $3.08 \pm 0.09 \mathrm{a}$ & $3.76 \pm 0.05 \mathrm{~d}$ \\
\hline Técnica de vinificación & & & \\
\hline MT & $12.23 \pm 0.57 \mathrm{~ns}$ & $2.70 \pm 0.21 \mathrm{~ns}$ & $3.88 \pm 0.18 \mathrm{~ns}$ \\
EDA + ME & $12.27 \pm 0.52 \mathrm{~ns}$ & $2.69 \pm 0.21 \mathrm{~ns}$ & $3.91 \pm 0.17 \mathrm{~ns}$ \\
\hline
\end{tabular}

Tabla 2. Valores medios \pm desviaciones estándar de los índices de color de los vinos. Los valores seguidos por una misma letra no presentaron diferencias en el test de Tukey al $5 \%$.

\begin{tabular}{|cccccc|}
\hline & Intensidad colorante & Tonalidad & $\mathbf{L}^{*}$ & $\mathbf{a}^{*}$ & $\mathbf{b}^{*}$ \\
\hline Variedad & & & & & \\
\hline Syrah & $4.93 \pm 0.29 \mathrm{~cd}$ & $0.73 \pm 0.03 \mathrm{c}$ & $71.8 \pm 1.6 \mathrm{~b}$ & $26.7 \pm 2.1 \mathrm{~d}$ & $-0.4 \pm 1.5 \mathrm{e}$ \\
Cab. Franc & $5.32 \pm 0.54 \mathrm{c}$ & $0.84 \pm 0.04 \mathrm{~b}$ & $71.5 \pm 2.5 \mathrm{~b}$ & $25.1 \pm 2.9 \mathrm{e}$ & $5.6 \pm 1.7 \mathrm{bc}$ \\
Merlot & $5.72 \pm 0.35 \mathrm{c}$ & $0.71 \pm 0.01 \mathrm{c}$ & $69.2 \pm 1.5 \mathrm{~b}$ & $29.5 \pm 1.7 \mathrm{c}$ & $4.2 \pm 0.5 \mathrm{c}$ \\
Cab. Sauv. & $4.56 \pm 0.35 \mathrm{~d}$ & $0.95 \pm 0.05 \mathrm{a}$ & $75.7 \pm 1.9 \mathrm{a}$ & $18.3 \pm 1.9 \mathrm{f}$ & $7.1 \pm 1.1 \mathrm{~b}$ \\
Marselan & $11.25 \pm 0.53 \mathrm{a}$ & $0.92 \pm 0.02 \mathrm{a}$ & $49.4 \pm 1.2 \mathrm{c}$ & $35.8 \pm 1.4 \mathrm{~b}$ & $10.5 \pm 1.0 \mathrm{a}$ \\
Tannat & $9.67 \pm 0.75 \mathrm{~b}$ & $0.70 \pm 0.04 \mathrm{c}$ & $52.3 \pm 2.7 \mathrm{c}$ & $40.7 \pm 2.7 \mathrm{a}$ & $2.5 \pm 1.3 \mathrm{~d}$ \\
\hline Técnica de vinificación & & & & & \\
\hline MT & $7.11 \pm 2.69 \mathrm{~ns}$ & $0.79 \pm 0.09 \mathrm{~ns}$ & $64.0 \pm 10.4 \mathrm{~ns}$ & $30.6 \pm 7.2 \mathrm{~ns}$ & $4.1 \pm 3.6 \mathrm{~ns}$ \\
EDA + ME & $6.71 \pm 2.64 \mathrm{~ns}$ & $0.83 \pm 0.12 \mathrm{~ns}$ & $65.9 \pm 10.8 \mathrm{~ns}$ & $28.2 \pm 8.0 \mathrm{~ns}$ & $5.8 \pm 3.6 \mathrm{~ns}$ \\
\hline
\end{tabular}

óptico para los análisis de color y celdas de vidrio de $1 \mathrm{~cm}$ para los análisis de polifenoles.

El análisis estadístico se hizo empleando Statgraphics Centurión XVI.II (StatPoint Technologies, USA).

Se hicieron análisis de varianza multifactoriales y separaciones de medias mediante Tukey al 5\%, contrastando por un lado los vinos de las distintas variedades y por otro los vinos elaborados con las dos técnicas alternativas.

Se hizo un Análisis Discriminante, empleando las variables que definen el color y la composición polifenólica de los vinos.

\section{Resultados y discusión}

La composición básica de los vinos de las distintas variedades en los dos años se muestra en la Tabla 1.

No se observó un impacto importante de las dos técnicas alternativas de vinificación en la composición básica de los vinos. En cambio, hubo diferencias significativas entre los vinos de las distintas variedades.

Todos los vinos tuvieron valores de $\mathrm{pH}$ elevados, siendo en algunos casos excesivamente altos (Cabernet Franc, Marselan y Cabernet Sauvignon), lo que puede ocasionar problemas de conservación y estabilidad [2].

El grado alcohólico más elevado lo tuvieron los vinos Cabernet Franc, Marselan y Tannat, en tanto el más bajo lo presentaron los vinos Cabernet Sauvignon (Tabla 1).
Los vinos de las distintas variedades presentaron diferencias significativas en el color. La mayor intensidad colorante fue presentada por los vinos marselan y la menor por los vinos Cabernet Sauvignon (Tabla 2). Los vinos más claros fueron los Syrah y los más oscuros los Marselan (menores valores de $\mathrm{L}^{*}$ ).

Los valores medios de los índices de color no presentaron diferencias significativas entre las técnicas de vinificación (Tabla 2). Estos resultados no coinciden con lo expresado por Bosso et al. [8,9] que obtuvieron una mejora del color con la extracción diferida de antocianos, pero concuerdan con lo reportado por trabajos previos realizados con Tannat, en los que se verificó que la extracción de estas moléculas fue disminuida cuando no se realizaron movimientos en la masa de orujos desde el principio de la maceración $[16,17]$. A su vez, numerosos autores verificaron que las maceraciones largas determinan que el color de los vinos tenga menor intensidad y sea más claro que el obtenido con maceraciones cortas $[7,18]$.

Los valores de tonalidad de todos los vinos fueron demasiado altos para su edad, mostrando una evolución del color, que podría atribuirse a procesos de oxidación importantes (Tabla 2). Este fenómeno se podría haberse dado particularmente en Marselan y Cabernet Sauvignon, incentivado por la baja acidez de esos vinos.

Los contenidos fenólicos de los vinos producidos con cada variedad presentaron diferencias significativas (Tabla 3). Los mayores contenidos fenólicos totales y 
Tabla 3. Valores medios \pm desviaciones estándar de la composición polifenólica de los vinos. Los valores seguidos por una misma letra no presentaron diferencias en el test de Tukey al 5\%.

\begin{tabular}{|crrcc|}
\hline & Polifenoles totales & Antocianos & Catequinas & Proantoc. \\
\hline Variedad & & & \\
\hline Syrah & $690 \pm 25 \mathrm{c}$ & $222 \pm 7 \mathrm{~d}$ & $422 \pm 33 \mathrm{~d}$ & $877 \pm 82 \mathrm{~d}$ \\
Cab. Franc & $733 \pm 42 \mathrm{c}$ & $256 \pm 25 \mathrm{c}$ & $404 \pm 71 \mathrm{~d}$ & $957 \pm 91 \mathrm{~d}$ \\
Merlot & $1134 \pm 53 \mathrm{~b}$ & $253 \pm 31 \mathrm{c}$ & $884 \pm 117 \mathrm{~b}$ & $1539 \pm 148 \mathrm{c}$ \\
Cab. Sauv. & $1165 \pm 34 \mathrm{~b}$ & $320 \pm 24 \mathrm{~b}$ & $1120 \pm 119 \mathrm{a}$ & $1551 \pm 141 \mathrm{c}$ \\
Marselan & $1220 \pm 99 \mathrm{~b}$ & $385 \pm 26 \mathrm{a}$ & $737 \pm 97 \mathrm{c}$ & $1810 \pm 78 \mathrm{~b}$ \\
Tannat & $1325 \pm 42 \mathrm{a}$ & $375 \pm 21 \mathrm{a}$ & $862 \pm 34 \mathrm{~b}$ & $2022 \pm 55 \mathrm{a}$ \\
\hline Técnica de vinificación & & & & \\
\hline MT & $1015 \pm 246 \mathrm{~b}$ & $320 \pm 67 \mathrm{a}$ & $677 \pm 249 \mathrm{~b}$ & $1455 \pm 422 \mathrm{~ns}$ \\
EDA + ME & $1074 \pm 259 \mathrm{a}$ & $283 \pm 63 \mathrm{~b}$ & $800 \pm 284 \mathrm{a}$ & $1463 \pm 453 \mathrm{~ns}$ \\
\hline
\end{tabular}

de proantocianidinas se obtuvieron en los vinos Tannat, los mayores contenidos de antocianos en Marselan y Tannat, y los mayores contenidos de catequinas en Cabernet Sauvignon. Estos resultados sugieren que las características propias de las uvas de cada variedad tienen una incidencia preponderante en la composición de los vinos, más allá de las técnicas de vinificación empleadas.

Las alternativas de vinificación empleadas modificaron significativamente la composición polifenólica de los vinos. Los contenidos de antocianos fueron significativamente menores en los vinos obtenidos por EDA + ME que en los testigos (Tabla 3). Esta menor concentración de antocianos puede estar asociada a su participación en pigmentos poliméricos, ya que según algunos autores la polimerización se da sobre todo en la fase post-fermentativa de la maceración [7]. Sin embargo, la maceración extendida también aumenta las probabilidades de que muchas de las moléculas de antocianos sean retenidas por las partes sólidas de la uva o precipitadas en las borras al ser adsorbidas por las levaduras [5,6,18]. En ensayos previos se verificaron menores contenidos de antocianos en vinos de uvas Tannat vinificadas por EDA [16,17], atribuyéndose estos resultados a algunas características de esta variedad, como la baja extractibilidad de los antocianos [19], que justifica realizar operaciones que incentiven la solubilización de estos compuestos desde el inicio de las maceraciones para poder explotar mejor el potencial de las uvas $[1,19]$.

La extracción diferida con maceración extendida modificó de manera diferente los contenidos de taninos de bajo peso molecular de los vinos. Durante la extracción diferida habría una actividad pectolítica importante en los hollejos, que incidiría en la extracción posterior de taninos [9]. Sin embargo, los contenidos medios de proantocianidinas no presentaron diferencias entre los vinos obtenidos con ambas técnicas, lo que podría deberse a una refijación importante de los polifenoles en las partes sólidas de la uva durante las maceraciones extendidas.

En las primeras horas de la fermentación se extraen sobre todo taninos de bajo peso molecular desde los hollejos, dada su solubilidad en un medio acuoso [20]. Las moléculas de taninos de mayor tamaño están ligadas a otras macromoléculas, como polisacáridos y proteínas, por lo que la presencia de etanol es necesaria para
Tabla 4. Valores medios \pm desviaciones estándar de la reactividad de los taninos y sus índices de polimerización en los vinos. Los valores seguidos por una misma letra no presentaron diferencias en el test de Tukey al 5\%.

\begin{tabular}{|ccc|}
\hline & DMACH & DMACH/LA \\
\hline Variedad & \\
\hline Syrah & $42.0 \pm 2.4 \mathrm{c}$ & $48.4 \pm \mathrm{bc}$ \\
Cab. Franc & $46.3 \pm 6.5 \mathrm{c}$ & $48.4 \pm \mathrm{bc}$ \\
Merlot & $82.3 \pm 8.1 \mathrm{~b}$ & $53.6 \pm \mathrm{b}$ \\
Cab. Sauv. & $97.6 \pm 9.2 \mathrm{a}$ & $63.8 \pm \mathrm{a}$ \\
Marselan & $80.8 \pm 5.7 \mathrm{~b}$ & $44.6 \pm \mathrm{cd}$ \\
Tannat & $80.2 \pm 2.7 \mathrm{~b}$ & $39.6 \pm \mathrm{d}$ \\
\hline Técnica de vinificación & & \\
\hline MT & $66.9 \pm 20.0 \mathrm{~b}$ & $46.1 \pm \mathrm{b}$ \\
EDA + ME & $76.2 \pm 22.1 \mathrm{a}$ & $53.4 \pm \mathrm{a}$ \\
\hline
\end{tabular}

su extracción [20]. De esta manera un menor contacto de los hollejos con el líquido en los primeros días de la extracción diferida determinaría que se disuelvan menor cantidad de los taninos hidrosolubles, lo que daría resultados opuestos a los encontrados. Por el contrario, una maceración extendida incrementa la extracción de taninos de las semillas, de menor tamaño que los taninos de los hollejos $[21,22]$, independientemente de la madurez de la uva $[7,18]$. Esto indicaría que el efecto de la maceración prolongada sobre la composición tánica es más relevante que el de la extracción diferida, explicando los resultados obtenidos (Cuadro 3 y Fig. 2). En concordancia, diversos autores indican que el grado medio de polimerización de los taninos decrece en las maceraciones extendidas $[18,23]$, si bien otros autores indican que en las maceraciones muy largas (más de 20 días) también aumentan las concentraciones de los polímeros de cadena más larga, igualmente extraídos desde las semillas [7].

Algunos autores indican que la extracción de taninos desde hollejos y semillas es diferente según la madurez de la uva [18]; otros señalan que el grado de madurez de las uvas incide más en la tipicidad de los vinos que el tiempo de maceración [23]; finalmente, otros autores indican que la estructura polifenólica de los vinos depende más de la variedad de uva que de la técnica de vinificación [24]. 


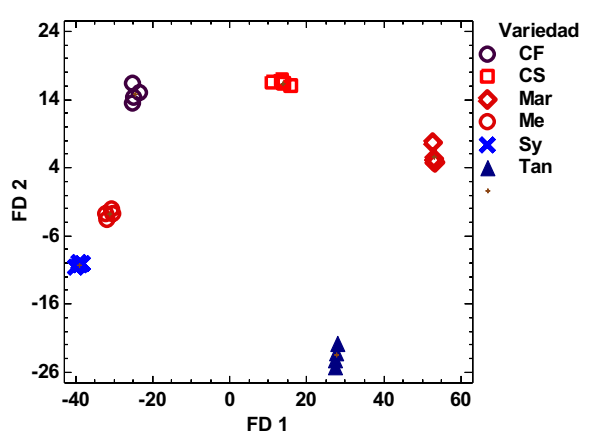

Figura 1. Distribución de las muestras de cada variedad en el plano generado por las dos primeras Funciones Discriminantes. $\mathrm{CF}=$ Cabernet Franc, $\mathrm{CS}=$ Cabernet Sauvignon, Mar $=$ Marselan, $\mathrm{Me}=$ Merlot, $\mathrm{Sy}=$ Syrah, Tan $=$ Tannat.

El Análisis Discriminante, realizado con las variables que definen el color y la composición fenólica de los vinos, permitió separar todas las muestras de acuerdo con la variedad (Fig. 1).

Los resultados obtenidos indican que el factor varietal fue el que tuvo mayor incidencia en el color y composición de los vinos, más allá de los efectos determinados por las alternativas de vinificación empleadas.

\section{Conclusiones}

Los vinos de las distintas variedades presentaron diferencias importantes en su composición y color.

Los vinos Tannat y Marselan tuvieron intensidades colorantes y contenidos fenólicos significativamente mayores a los de las otras variedades.

Las alternativas de vinificación empleadas determinaron diferencias en la composición fenólica de los vinos de las distintas variedades, y sobre todo en la estructura tánica de los mismos, sin diferencias estadísticas en el color.

La vinificación por EDA + ME determinó menores contenidos de antocianos y mayores contenidos de polifenoles totales y catequinas. El empleo conjunto de EDA + ME disminuyó el grado medio de polimerización de los taninos.

Estos resultados indican que la evolución del color de los vinos puede ser condicionada de manera diferente por las alternativas de vinificación empleadas, por lo que sería interesante continuar la evaluación durante un tiempo más prolongado.

La variedad de uva fue el factor que incidió en mayor medida en las características de los vinos, más allá de las diferencias determinadas por las técnicas de vinificación empleadas en cada caso.

El presente trabajo fue financiado por la Agencia Nacional de Investigación e Innovación (A.N.I.I.), a través del proyecto FMV_2_2011_1_6332.

Los autores agradecen a Cecilia Baldi, Natalia Hernández, Sofía Traverso, María José Dodero, Valentina Peirano y Marcelo Breganti, por su participación en distintas etapas de este trabajo.
Los análisis de rutina de los vinos fueron realizados en el Instituto Nacional de Vitivinicultura.

\section{Referencias}

[1] K. Sacchi, L. Bisson, D. Adams, Am. J. Enol. Vitic. 56, 197 (2005)

[2] V. Cheynier, M. Dueñas-Paton, E. Salas, C. Maury, J.M. Souquet, P. Sarni-Manchado, H. Fulcrand, Am. J. Enol. Vitic. 57, 298 (2006)

[3] H. Fulcrand, M. Dueñas, E. Salas, V. Cheynier, Am. J. Enol. Vitic. 57, 289 (2006)

[4] R. Canals, M. Llaudy, J. Valls, J. Canals, F. Zamora, J. Agric. Food Chem. 53, 4019 (2005)

[5] E. Gómez-Plaza, R. Gil-Muñoz, J. López-Roca, A. Martínez, J. Agric. Food Chem. 48, 736 (2000)

[6] A. Zimman, A. Waterhouse, Am. J. Enol. Vitic. 55, 139 (2002)

[7] L.F. Casassa, C.W. Beaver, M.S. Mireles, J.F. Harbertson, Aust. J. Grape Wine Res. 19, 25 (2013)

[8] A. Bosso, M. Guaita, L. Panero, D. Borsa, R. Di Stefano, XXVIII Congreso Mundial de la Viña y el Vino (2004)

[9] A. Bosso, M. Guaita, L. Panero, D. Borsa, R. Follis, Am. J. Enol. Vitic. 60, 379 (2009)

[10] Y. Glories, Conn. Vigne Vin 18, 253 (1984)

[11] F. Ayala, J.F. Echávarri, A.I. Negueruela, Am. J. Enol. Vitic. 48, 357 (1997)

[12] V. Singleton, J. Rossi, Am. J. Enol. Vitic. 16, 144 (1965)

[13] P. Ribéreau-Gayon, E. Stonestreet, Bull. Soc. Chim. 9, 2649 (1965)

[14] T. Swain, W. Hillis, J. Sci. Food Agric. 10, 63 (1959)

[15] P. Ribéreau-Gayon, E. Stonestreet, Chim. Anal. 48, 188 (1966)

[16] G. González-Neves, G. Gil, L. Barreiro, R. Bochicchio, G. Gatto, A. Tessore, G. Favre, J. Food Comp. Anal. 23, 447 (2010)

[17] G. González-Neves, G. Gil, G. Favre, M. Ferrer, Int. J. Food Sci. Technol. 47, 900 (2012)

[18] M. Gil, N. Kontoudakis, E. Gonzalez, M. Esteruelas, F. Fort, J.M. Canals, F. Zamora, J. Agric. Food Chem. 60, 7988 (2012)

[19] G. González-Neves, D. Charamelo, J. Balado, Barreiro, R. Bochicchio, G. Gatto, G. Gil, L.A. Tessore, A. Carbonneau, M. Moutounet, Anal. Chim. Acta 513, 191 (2004)

[20] C. Morel-Salmi, J.M. Souquet, M. Bes, V. Cheynier, J. Agric. Food Chem. 54, 4270 (2006)

[21] F. Brossaud, V. Cheynier, A. Noble, Aust. J. Grape Wine Res. 7, 33 (2001)

[22] M. Downey, J. Harvey, S. Robinson, Aust. J. Grape Wine Res. 9, 15 (2003)

[23] Y. Cadot, S. Caillé, A. Samson, G. Barbeau, V. Cheynier, Anal. Chim. Acta 732, 91 (2012)

[24] G. Gambacorta, D. Antonacci, S. Pati, M. La Gatta, M. Faccia, A. Coletta, E. La Notte, Eur. Food Res. Technol. 233, 1057 (2011) 